\title{
Structure and biological activity analysis of fucoidan isolated from
}

\section{Sargassum siliquosum}

Shao-Hua Wang ${ }^{1}$, Chih-Yu Huang ${ }^{1,2}$, Chun-Yen Chen ${ }^{3}$, Chia-Che Chang ${ }^{4,5,6}$, Chun-

Yung Huang 7 , Cheng-Di Dong ${ }^{2 * *}$, and Jo-Shu Chang ${ }^{1,8,9^{*}}$

${ }^{1}$ Department of Chemical Engineering, National Cheng Kung University, Tainan,

Taiwan

${ }^{2}$ Department of Marine Environmental Engineering, National Kaohsiung University of

Science and Technology, Nanzih District, Kaohsiung, Taiwan

${ }^{3}$ University Center for Bioscience and Biotechnology, National Cheng Kung

University, Tainan, Taiwan

${ }^{4}$ Institute of Biomedical Science, National Chung Hsing University, Taichung,

Taiwan

${ }^{5}$ Traditional Herbal Medicine Research Center, Taipei Medical University Hospital,

Taipei, Taiwan

${ }^{6}$ Department of Medical Research, China Medical University Hospital, Taichung,

Taiwan

${ }^{7}$ Department of Seafood Science, National Kaohsiung University of Science and

Technology, Nanzih District, Kaohsiung, Taiwan

${ }^{8}$ Department of Chemical and Materials Engineering, College of Engineering,

Tunghai University, Taichung, Taiwan

${ }^{9}$ Research Center for Smart Sustainable Circular Economy, Tunghai University, Taichung, Taiwan 


\section{Supporting Information}

HepG2 cells were stained with Oil Red treatment after free fatty acid (FFA) induced lipid accumulations. Anti-lipogenesis activity of crude extract (a) and fucoidan (b) from S. siliquosum were tested by treating HepG2 cells through Oil Red coloration revealed of lipid formation concentrations (Figure S1).
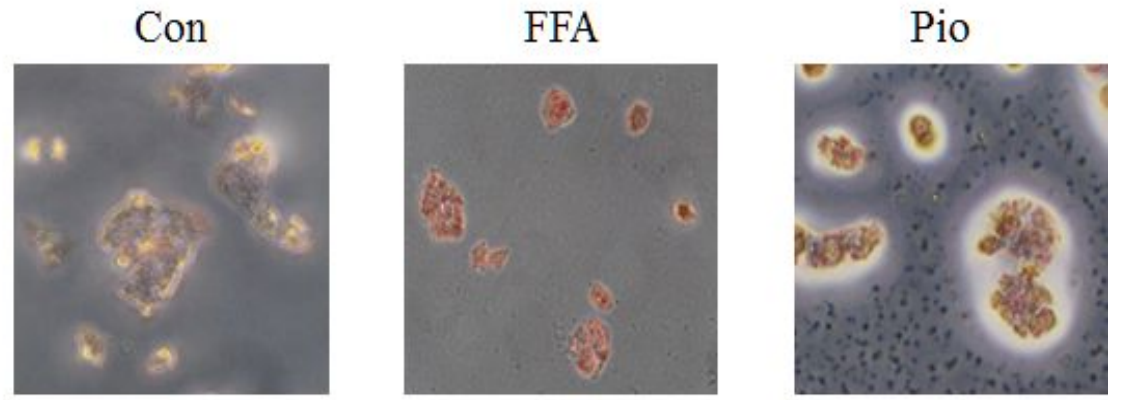

(a)
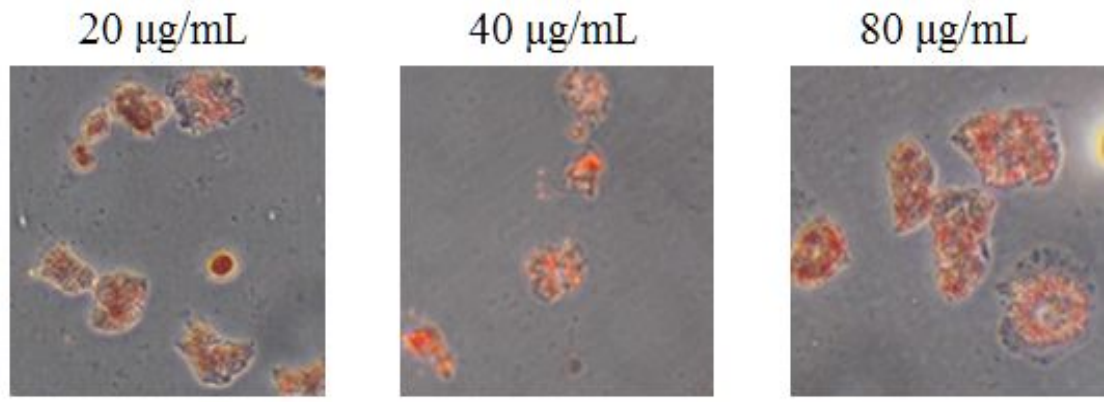

(b)
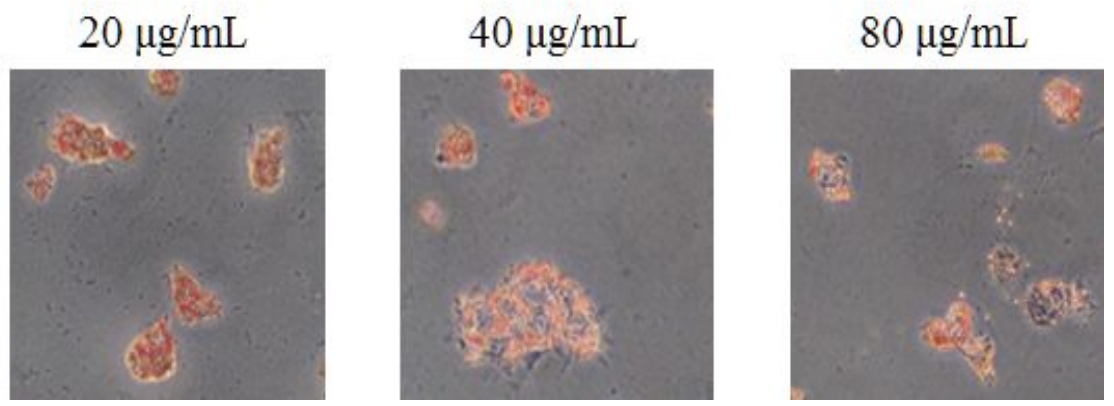

Figure S1. Anti-lipogenesis activity of crude extract (a) and fucoidan (b) from $S$. 
siliquosum. Cells were stained with Oil Red treatment. Con: Without free fatty acidtreated control cells; FFA: free fatty acid-treated cells; Pio: The clinical drug pioglitazone used for the treatment of diabetes was used as a positive control for testing lipid content decreased by co-treatment with free fatty acid. 